\title{
Two-stage Multivariable IMC Antiwindup (TMIA) control of a Quadruple Tank Process using a PLC
}

Link to publication record in Manchester Research Explorer

\section{Citation for published version (APA):}

King-Hans, A. R., Heath, W. P., \& Alli-Oke, R. (2014). Two-stage Multivariable IMC Antiwindup (TMIA) control of a Quadruple Tank Process using a PLC. In IEEE Multi-conference on Systems and Control (pp. 1681-1686). IEEE.

\section{Published in:}

IEEE Multi-conference on Systems and Control

\section{Citing this paper}

Please note that where the full-text provided on Manchester Research Explorer is the Author Accepted Manuscript or Proof version this may differ from the final Published version. If citing, it is advised that you check and use the publisher's definitive version.

\section{General rights}

Copyright and moral rights for the publications made accessible in the Research Explorer are retained by the authors and/or other copyright owners and it is a condition of accessing publications that users recognise and abide by the legal requirements associated with these rights.

\section{Takedown policy}

If you believe that this document breaches copyright please refer to the University of Manchester's Takedown Procedures [http://man.ac.uk/04Y6Bo] or contact uml.scholarlycommunications@manchester.ac.uk providing relevant details, so we can investigate your claim.

\section{OPEN ACCESS}




\title{
Two-stage Multivariable IMC Antiwindup (TMIA) control of a Quadruple Tank Process using a PLC
}

\author{
Awo R. King-Hans, William P. Heath, Razak Alli-Oke
}

\begin{abstract}
Actuator saturation is a common phenomenon in the control of multivariable systems which causes two major problems for control engineers, namely windup and directionality. This paper focuses on a Two-stage Multivariable IMC Antiwindup (TMIA) structure for open-loop stable plants. This IMC-based control structure is of interest because it tackles the aforementioned problems in an intuitive and easy to tune way. The highlight of this structure is the solution of two low-order quadratic programs to control both steady-state and transient behaviours of the plant. The controller is tested by application to a multivariable Quadruple Tank process controlled by a PLC. The TMIA structure is found to outperform its IMC counterparts in handling windup and directionality. Results obtained demonstrate the realizability of the advanced control technique on an off-the-shelf industrial PLC. Thus the TMIA structure is presented as a competitive alternative in terms of tuning transparency and reduced computations to other advanced control techniques such as MPC which are limited by the low computational power offered by standard PLCs.
\end{abstract}

\section{INTRODUCTION}

All real world control applications involve magnitude or rate constraints on actuators. If these constraints are not accounted for in the controller design, it could lead to poor transient response, closed-loop performance degradation and even instability [1]. At these constraint limits, saturation occurs and there is a mismatch between the requested controller output and the achievable plant input. The significant difference causes an accumulation of error which must be offset in the opposite direction long enough for the controller action to return to normal. This results in transients/overshoots which must decay before the system returns to a linear regime and the controller is said to 'windup'. In the design of analytical dynamic controllers such as Internal Model Control, a common approach is to first design a linear controller neglecting the constraints which satisfies all the closed-loop performance requirements and then add a saturation compensation scheme to ensure graceful performance degradation of the closed-loop when the constraints become active. These ad hoc methods are usually known as antiwindup compensation [1]-[4].

In multivariable systems, a further problem associated with actuator saturation is called input directionality. Unlike SISO systems, the MIMO gain depends on the direction of the input vector [5]. A process exhibits directionality when the

The authors are with the Control Systems Centre, School of Electrical and Electronic Engineering, The University of Manchester, Manchester, M13 9PL, UK.

awo.king-hans@manchester.ac.uk

william.heath@manchester.ac.uk

razak.alli-oke@manchester.ac.uk saturated controller output yields a system response that is not 'closest' to the system response of the unconstrained controller output [6]. The problem of directionality compensation is calculating a feasible plant input on the basis of a given unconstrained controller output. In [6], [7], an optimal directionality compensation problem is presented as a finite-time horizon, state dependent, constrained quadratic optimization problem. Its objective is to minimize the distance between the output of the unsaturated plant with an ideal controller and the output of the saturated plant with a directionality compensator. This problem is similarly dealt with under the name of input allocation with an extensive survey in [8] on control allocation algorithms for linear and non-linear models.

The problems of windup and directionality can be overcome with Model Predictive Control where the constraints are explicitly accounted for and the controller action is the solution to a constrained optimization problem [7]. MPC applications have gained widespread use in industrial process especially in refining and petrochemical industries [9]. However, robust MPC design techniques increase the controller computational and memory requirements and long-horizon MPC is usually deployed on expensive dedicated digital computers. There is current research into fast online MPC implementation [10]-[13] on platforms such as Programmable Logic Controllers (PLCs) and Programmable Automation Controllers (PACs) [14]. A novel IMC-based online optimizing anti-windup control structure that simultaneously handles the issues of windup and directionality is proposed in [15]. This Two-stage Multivariable IMC Antiwindup (TMIA) control structure does not require the receding horizon computation of MPC and may serve as an alternative to MPC which is computationally less expensive and more transparent in terms of tuning and robustness. This is particularly attractive because it can be implemented on a cheap digital industrial platform as we demonstrate in this paper. Simulation results presented in [15] show that the TMIA controller competes favourably with long horizon MPC (where the number of optimization variables and constraints is a multiple of the prediction horizon [8]) while only requiring the computation capacity of a single horizon MPC.

This paper focuses on real-time implementation of IMC antiwindup strategies on a PLC for the control of a Quadruple Tank system described in [16]. It compares the TMIA controller with other IMC strcutures in terms of performance and highlights the computation requirements for the implementation of this optimization-based algorithm on a PLC. 


\section{Two stage Multivariable IMC Antiwindup (TMIA) CONTROL FORMULATION}

A classical IMC design [17] consists of two major steps: model factorization and robustness filter design where the plant model $\tilde{G}(z)$ is factorized into an invertible and noninvertible part such that $\tilde{G}(z)=\tilde{G}_{+}(z) \tilde{G}_{-}(z)$ and $\tilde{G}_{+}(1)=I$. $\tilde{G}_{+}(z)$ contains time delays and zeros of $\tilde{G}(z)$ outside the complex unit circle and $\tilde{G}_{-}(z)$ has a stable realizable inverse. Addition of a low-pass filter $F(z)$ provides robustness of the IMC loop to modelling errors. For a multivariable plant, a diagonal filter is used and the IMC controller is given by

$$
\begin{gathered}
Q_{I M C}(z)=\tilde{G}_{-}(z)^{-1} F(z) . \\
F(z)=\operatorname{diag}\left(\frac{1-a_{i}}{1-a_{i} z^{-1}}\right), \quad 0 \leq a_{i} \leq 1 \quad i=1,2, \ldots, m,
\end{gathered}
$$

where $m$ is the number of manipulated variables. Adjustment of the filter tuning parameter $a_{i}$ is very transparent and suitable for manipulating online. This makes IMC a good choice for multivariable control.

A modified IMC structure shown in Figure 1 is described in [2] to deal with the undesirable effects associated with the standard IMC structure during saturation. $Q_{I M C}(z)$ is assumed to be bi-proper minimum phase stable and is factorized as shown in Figure 1 such that:

$$
Q_{I M C}=\left(I+Q_{b}(z)\right)^{-1} Q_{f}(z) .
$$

$F_{B}(z)$ is an additional filter discussed in Section III-C. Assuming no plant-model mismatch, the closed-loop equations are given by

$$
\begin{aligned}
& u(z)=Q_{f}(z) F_{B}(z)(r(z)-\tilde{d}(z))-Q_{b}(z) v(z) \\
& y(z)=G(z) v(z)+d(z)
\end{aligned}
$$

where the controller (4) is directly fed with information on the saturation action. One choice of $Q_{f}(z)$ and $Q_{b}(z)$ [2] is

$$
\begin{aligned}
Q_{f}(z) & =F_{A}(z) \tilde{G}(z) Q_{I M C}(z) \\
Q_{b}(z) & =F_{A}(z) \tilde{G}(z)-I
\end{aligned}
$$

where the non-causal filter $F_{A}(z)$ is chosen to be diagonal in order to avoid introducing any change in the output direction and such that $\lim _{z \rightarrow \infty}\left[F_{A}(z) \tilde{G}(z)\right]=I . Q_{f}(z)$ is minimum phase stable to guarantee internal stability of the closed-loop system and $Q_{b}(z)$ must be strictly proper to be implementable (free of algebraic loops).

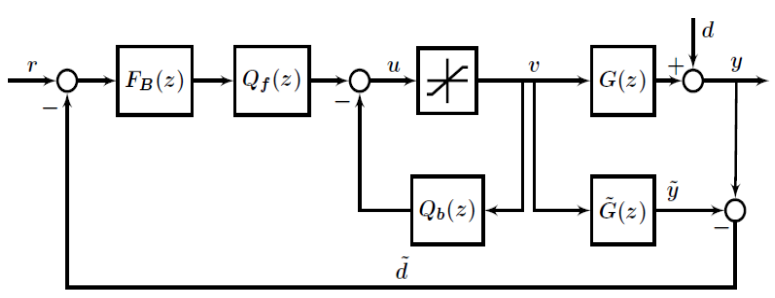

Fig. 1: Modified IMC Antiwindup Structure

A novel TMIA control structure proposed in [15] directly addresses the problems of windup and directionality change which affect multivariable plants under input constraints such as actuator saturation. Two low-order Quadratic Programs $(\mathrm{QPs})$ are solved at each sample step: the dynamic QP $\left(Q P_{1}\right)$ shown in Figure 2 addresses transient behaviour of the plant ensuring that the constrained system response is as close as possible to the unconstrained, while the steady-state QP $\left(Q P_{2}\right)$ ensures optimal steady-state performance. The two

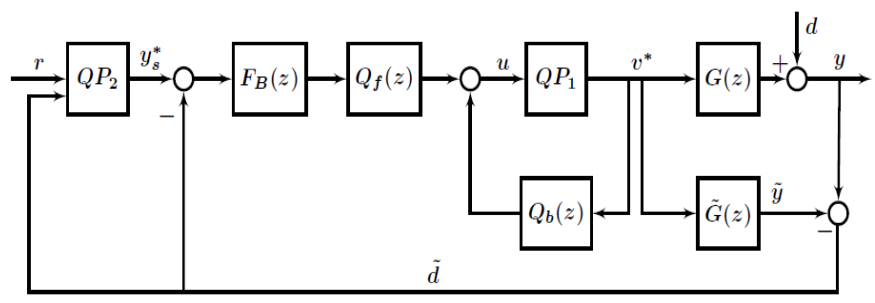

Fig. 2: TMIA Control Structure

QPs have similar structures - convex QPs solved over a single-step horizon subject to the same input constraints. For the dynamic QP, the optimization problem is formulated as:

$$
\begin{gathered}
v^{*}=\underset{v}{\arg \min }\|C v-C u\|_{W}^{2} \\
\text { subject to } \quad u_{i}^{\min } \leq v_{i} \leq u_{i}^{\max } \quad i=1, \ldots, m .
\end{gathered}
$$

where $m$ is the number of manipulated variables and $v$ and $u$ are the constrained and unconstrained inputs respectively with $W$ assumed to be a positive definite symmetric matrix that penalizes deviations in the constrained control inputs from the unconstrained and their relative importance. $C$ is the characteristic matrix of the square system as defined as $\lim _{z \rightarrow \infty}\left[\operatorname{diag}\left\{z_{m}^{r}\right\} G(z)\right]$. The steady-state performance optimization seeks to minimize the error between feasible steadystate target $y_{s}$ and the reference $r$ within the limitations of the input constraints by obtaining suitable values for $y_{s}$ and $u_{s}$ which satisfies:

$$
y_{s}=K_{p} u_{s}+\tilde{d}
$$

where $u_{s}$ is the value of the control input which causes the controlled variable to attain $y_{s}$ in steady-state. From Figure 2, $\tilde{d}$ is the disturbance estimate obtained as the difference between the measured plant output $y$ and the model output $\tilde{y}$ and $K_{p}$ is the steady-state gain of the plant $(G(1)$ for discretetime systems). The steady-state QP is solved to obtain an optimal feasible steady-state target $y_{s}^{*}$ according to:

$$
y_{s}^{*}=\underset{u_{s}, y_{s}}{\arg \min }\left\|r-y_{s}\right\|_{Q_{s s}}^{2}
$$

subject to the inequality and equality constraints:

$$
u_{i}^{\min } \leq u_{s_{i}} \leq u_{i}^{\max } \quad i=1, \ldots, m \quad\left[\begin{array}{ll}
-K_{p} & I
\end{array}\right]\left[\begin{array}{l}
u_{S} \\
y_{s}
\end{array}\right]=\tilde{d}
$$

where $Q_{s s}$ is a positive definite symmetric matrix for penalizing deviations in the controlled variables and their relative importance. The equality constraint ensures the steady-state requirement of (9).

The TMIA structure summarily works such that if the set point target is achievable in steady-state, the difference 
between the reference $r$ and the disturbance estimate $\tilde{d}$ is passed to the dynamic QP for the solution of the optimal control input $v^{*}$. Otherwise if input constraint violation causes the steady-state target to be unachievable, the steadystate QP computes a feasible steady-state target $y_{s}$ which is optimally close to reference $r$. The difference between the feasible steady-state target and the current disturbance estimate is then passed to the dynamic QP for computation of $v^{*}$.

An equivalent optimization problem to (10) can be obtained by eliminating the equality constraints to obtain:

$$
\begin{gathered}
\qquad u_{s}^{*}=\underset{u_{s}}{\arg \min }\left\|r-\tilde{d}-K_{p} u_{s}\right\|_{Q_{s s}}^{2} \\
\text { subject to } \quad u_{i}^{\min } \leq u_{s_{i}} \leq u_{i}^{\max } \quad i=1, \ldots, m
\end{gathered}
$$

This transformed problem is now to optimize only over variable $u_{s}$ with inputs as the difference between reference $r$ and the disturbance estimate $\tilde{d}$. An optimal solution $u_{s}^{*}$ of (11) ensures that the optimal $y_{s}^{*}$ of the original problem in (10) is found via the steady-state model of (9).

Equations (8) and (11) can be written in standard QP form as:

$$
\begin{gathered}
Q P_{1}: v^{*}=\underset{v}{\arg \min } \frac{1}{2} v^{T} H_{1} v-v^{T} \tilde{H}_{1} u \quad \text { subject to } L v \leq b \\
Q P_{2}: u_{s}^{*}=\underset{u_{s}}{\arg \min } \frac{1}{2} u_{s}^{T} H_{2} u_{s}-u_{s}^{T} \tilde{H}_{2}^{T}(r-\tilde{d})
\end{gathered}
$$$$
\text { subject to } L u_{s} \leq b \quad \text { and } \quad y_{s}^{*}=\tilde{H}_{2} u_{s}^{*}+\tilde{d}
$$

where $H_{1}=\tilde{H}_{1}{ }^{T} W \tilde{H}_{1}$ and $H_{2}=\tilde{H}_{2}{ }^{T} Q_{s s} \tilde{H}_{2}$ are symmetric positive definite Hessian matrices which are defined based on the structural properties of the plant such that $\tilde{H}_{1}$ and $\tilde{H}_{2}$ are the characteristic matrix and steady-state gain matrix of the plant respectively (i.e. $\tilde{H}_{1}=C$ and $\tilde{H}_{2}=K_{p}$ ). $L$ and $b$ in the inequality constraints are defined as:

$$
L=\left[\begin{array}{c}
-I_{m} \\
I_{m}
\end{array}\right] \quad \text { and } \quad b=\left[\begin{array}{c}
-u^{\min } \\
u^{\max }
\end{array}\right]
$$

\section{THE QUAdRUPLE TANK SET UP}

\section{A. Description}

The Quadruple Tank system is a multivariable laboratory process consisting of 4 interconnected water tanks of uniform cross-sectional area, 2 pumps and a water basin as presented in [16]. The Quadruple Tank system fabricated by Quanser [18] is shown in Figure 3 with inputs as voltages to the 2 pumps, and outputs as water levels in the two lower tanks.

The maximum operating voltage of the pumps is $12 \mathrm{~V}$, hence the inputs are constrained between $0-10 \mathrm{~V}$ in the controller design. Each of the pumps thrust water vertically to the tanks via two orifices of different sizes provided by Quanser. Through these orifices, a piping connection is made to the tank based on the configuration required. Each tank also has outlets for discharge of water back into the water basin and the user can also vary the areas of discharge using different orifice sizes. The apparatus thus forms an autonomous and closed recirculating system [18].

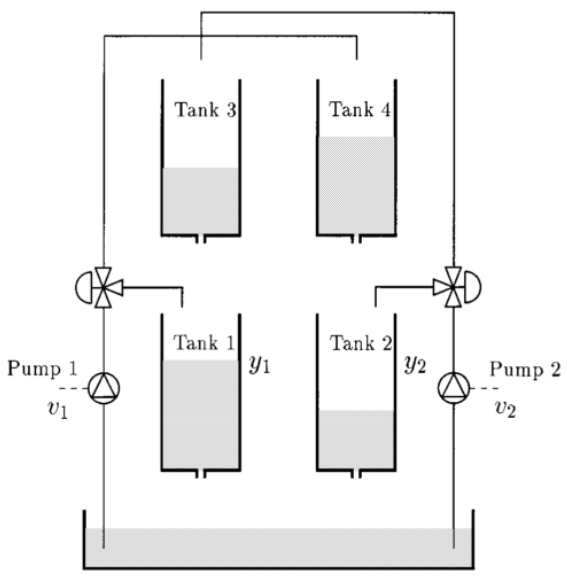

Fig. 3: Schematic diagram of the Quadruple Tank process

The Quadruple tank process can be set up to be minimum or non-minimum phase depending on the sizes of the orifices and piping connections used. Details of how the location of zeros inside or outside the unit circle can be adjusted by varying the valve positions is described in [16].

The water levels are measured by pressure sensors located at the bottom of each tank. The sensor output is a $0-5 \mathrm{~V}$ analog signal which corresponds to $0-25 \mathrm{~cm}$ of water height. This $0-5 \mathrm{~V}$ analog signal is interfaced with the PC through a signal conditioning board. The Quanser quadruple tank system is provided with hardware and QuaRC software that allows real-time interface between MATLAB its sensors and actuators. The complete real-time control of the Quadruple Tank process include hardware peripherals which are:

1) Quanser 8-channel USB Data Acquisition board,

2) Analog Sensors Adapter and

3) 2-channel Linear Voltage Amplifier.

Two control platforms are utilized; the QuaRC platform for testing the control design before deploying to a Siemens PLC which is main focus of our results.

This paper only reports results for the minimum phase setting of the quadruple tanks. This is desirable as the configuration allows higher closed-loop bandwidth and thus requires faster computation on the PLC. The TMIA controller is also suitable for non-minimum phase systems and has been implemented by carrying out an inner-outer factorization of the plant model but results are beyond the scope of this paper. The plant is calibrated according to information provided in the Quanser user manual [18]. Figure 4 shows a snapshot of the Quadruple Tank system connected to the hardware peripherals and PLC.

\section{B. Model Identification}

The system transfer function is of the form

$$
\left[\begin{array}{l}
y_{1} \\
y_{2}
\end{array}\right]=\left[\begin{array}{ll}
G_{11} & G_{12} \\
G_{21} & G_{22}
\end{array}\right]\left[\begin{array}{l}
v_{1} \\
v_{2}
\end{array}\right]
$$

where $y_{1}$ and $y_{2}$ are the levels of the two lower tanks and $v_{1}$ and $v_{2}$ are the input voltages from pumps 1 and 2 respectively. 


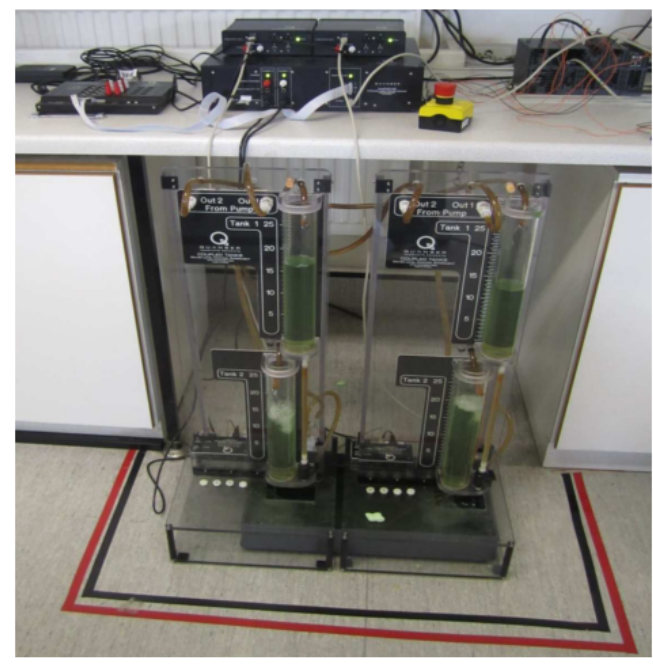

Fig. 4: Snapshot of the Quadruple Tank process with connections to a PLC and Quanser hardware. Top right corner is a Siemens SIMATIC S7-300 CPU 314C-2 PN/DP PLC. Top left corner is a Quanser Data Acquisition board. Top middle consists of Analog Sensors Adapter (above) and Voltage Amplifier (beneath).

A linearized system model was identified at an operating voltage of $[7 \mathrm{~V}, 7 \mathrm{~V}]$ with $1 \mathrm{~s}$-sampling as:

$$
\tilde{G}(z)=\left[\begin{array}{cc}
\frac{0.1359 z^{-2}}{1-0.9362 z^{-1}} & \frac{0.1326 z^{-2}-0.01253 z^{-3}}{1-0.3906 z^{-1}-0.5538 z^{-2}} \\
\frac{0.03781 z^{-2}-0.02813 z^{-3}}{1-1.833 z^{-1}-0.8395 z^{-2}} & \frac{0.1642 z^{-2}}{1-0.9215 z^{-1}}
\end{array}\right]
$$

The system is minimum phase stable with poles and zeros inside the unit circle.

\section{TMIA control design for Quadruple Tank}

A Two-stage Multivariable IMC Antiwindup controller is designed for the Quadruple Tank process. First, a classical IMC controller is designed factorizing the plant model $\tilde{G}(z)$ is into an invertible and non-invertible part.

$$
\tilde{G}_{-}(z)=\left[\begin{array}{cc}
\frac{0.1359}{1-0.9362 z^{-1}} & \frac{0.1326-0.01253 z^{-1}}{1-0.3906 z^{-1}-0.5538 z^{-2}} \\
\frac{0.03781-0.02813 z^{-1}}{1-1.833 z^{-1}-0.8395 z^{-2}} & \frac{0.1642}{1-0.9215 z^{-1}}
\end{array}\right]
$$

and $\tilde{G}_{+}(z)=z^{-2} I$, a robustness filter following from Equation (2) is designed as

$$
F(z)=\left[\begin{array}{cc}
\frac{1-0.65}{1-0.65 z^{-1}} & 0 \\
0 & \frac{1-0.85}{1-0.85 z^{-1}}
\end{array}\right]
$$

The filter $F(z)$ is used to avoid some chattering effects on the control signals [19] as well as shape the closed-loop response. For the Quadruple Tank system, the open-loop bandwidth is obtained approximately as $1 / \tau_{\max }$, where $\tau_{\max }$ is the maximum time constant of the system. The desired closed-loop bandwidth of the system is specified to be twice the open-loop bandwidth corresponding to about $0.2 \mathrm{rad} / \mathrm{s}$. An additional bandwidth filter $F_{B}(z)$ shown in Figure 2 is used to limit the cut-off frequency of the control signal to the closed-loop bandwidth.
The IMC tuning parameters, $\left(a_{1}=0.65, a_{2}=0.85\right)$ are such that the frequency of the maximum sensitivity is greater than the closed-loop bandwidth $(\bar{\sigma}(S)>0.2 \mathrm{rad} / \mathrm{s}) . F(z)$ is considered separately from $F_{B}(z)$ and is chosen such that $Q_{I M C}(z)$ is biproper according to [2] for the design of the MIMC controller. Also the specification for reference tracking and zero steady-state error is achieved since the conditions for integral action, $\left[F_{B}(z) F(z) G_{+}(z)\right]_{z \rightarrow 1}=I$ is met. The MIMC controller is now obtained by factorizing the biproper $Q_{I M C}(z)$ to obtain $Q_{b}(z)$ and $Q_{b}(f)$ using the non-causal filter $F_{A}(z)$ as

$$
F_{A}(z)=\left[\begin{array}{cc}
\frac{1}{0.1359} z & 0 \\
0 & \frac{1}{0.1642} z
\end{array}\right]
$$

such that $\lim _{z \rightarrow \infty}\left[F_{A}(z) \tilde{G}(z)\right]=I$. The characteristic and steady-state matrices for the Quadruple Tank are obtained as

$$
C=\left[\begin{array}{cc}
0.1359 & 0.1326 \\
0.03781 & 0.1642
\end{array}\right] \text { and } \quad K_{p}=\left[\begin{array}{cc}
2.13009 & 2.1595 \\
1.4892 & 2.0917
\end{array}\right]
$$

A primal-dual interior point method [20] is used for the quadratic optimization in the TMIA controller. The positive definite symmetric weighting matrices, $W$ and $Q_{s s}$ are chosen as identity. The stopping criterion for the interior point algorithm was set to $10^{-3}$ and the maximum number of iterations was constrained to 200 .

\section{Controller Implementation}

The TMIA control design was implemented in the Siemens SIMATIC S7-300 CPU 314C-2 PN/DP PLC using Ladder logic which is easily understood by operators in industry and as such easier to tune. The PLC consists of an integrated power supply and CPU unit and two I/O modules. The power supply converts $230 \mathrm{VAC}$ line voltage to a $24 \mathrm{VDC}$ operating voltage to supply its S7-300 rack and load circuits. The I/O modules consist of 24 digital inputs, 16 digital outputs, 5 analog inputs and 2 analog outputs. The inputs on the PLC side are the water levels in the two lower tanks while the outputs are the volage supplied to the pumps. Two analog $\mathrm{I} / \mathrm{Os}$ are used with a voltage range of $0-10 \mathrm{~V}$.

The PLC provides 192KB working memory on an external card. The CPU executes user programs and communicates with other nodes via an MPI interface which is an integrated interface for all Siemens SIMATIC products. The PLC also supports Ethernet communication via an integrated PROFINET (Industrial Ethernet) interface; this is the communication link used. The PLC is programmed using a Programming Device (PG) which in this case is a PC with Siemens Totally Integrated Automation (TIA) Portal version 11 software installed.

Real-time data acquisition is obtained via OPC toolbox and a KEPServerEX5 OPC server. With the controller running on the PLC, the KEPServerEX5 provides an interface for SIMULINK to read data continuously from PLC memory and to write desired set-points to the PLC. The PLC scan time is set to $1 \mathrm{~s}$ which coincides with the sampling time for the discretization of the plant transfer function. 


\section{COMPUTATIONAL CONSIDERATIONS}

The PLC program utilizes both memory and temporary variables. The values of the memory variables are stored in the PLC memory until a reset occurs while temporary variables are not stored. MD (Memory Double Word) variables are utilized in the TMIA program. The S7-300 CPU 314C2 PN/DP model provides 256 bytes of memory for the MD format which are 4 bytes long; this results in a total of 64 MD variables available for our use. Since this is insufficient for the TMIA program, temporary variables are utilized to a large extent. Only a few variables which need to be preloaded to memory such as the QP parameters and variables which need to be read by the OPC server are stored as MD variables. For example, the use of MD variables is limited by taking advantage of the symmetry of the Hessian matrices where only one off-diagonal element is stored in memory.

\section{RESULTS}

In order to test the efficiency of the TMIA controller, two set-point profiles were applied to the Quadruple Tank to sufficiently excite windup and process directionality in the system. Sufficient step magnitudes were chosen to simulate situations where the controllers to be compared would usually yield a degraded performance.

- Profile 1: Level set points of $12 \mathrm{~cm}$ applied in the same direction with no lag between them.

- Profile 2: Level set points of $2 \mathrm{~cm}$ applied in opposite directions with no lag between them.

The input voltages to the pumps are constrained between 0 and $10 \mathrm{~V}$ in the algorithm. The results of the PLC implementation of the TMIA controller are compared with classical IMC and MIMC structures as shown in Figures 5 and 6. The tank levels were allowed to settle for 250s in open-loop with the application of $(7 \mathrm{~V}, 7 \mathrm{~V})$ operating voltage before closedloop control was applied.

\section{A. Control Results}

1) Profile 1: In Figure 5 the IMC controller yields a poor performance due to saturation. This response is as expected since the IMC controller takes no account of the saturation effect and as such the output does not track setpoint when the constraints are active. The MIMC output yields a better performance where Tank 1 tracks the reference signal but Tank 2 is unable to meet the set-point due to active constraints. Since the TMIA algorithm works by recalculating an optimal realizable reference signal (not shown in figure for the sake of visual clarity), it recalculates a slightly higher set-point for Tank 1 and a lower one for Tank 2. The steadystate QP uses the knowledge of the constraints to calculate a new reference. It has been observed from simulation study that the TMIA algorithm avoids the constraint limits and only saturates where the requested step magnitude is very high as in this case. The weight $W$ in the QP parameters can be adjusted to reflect priority on a particular output in maintaining the set-point. It must also be noted that profile 1 requests set-points at the very limit of the tanks capacity which will not be the case in practice; this is in order to force the TMIA controller to saturation even though for a short time as seen in Figure 5b. For a reduced set-point than this value, the TMIA controller achieves perfect set-point tracking and the realizable reference is kept as close to the set-point at all times.

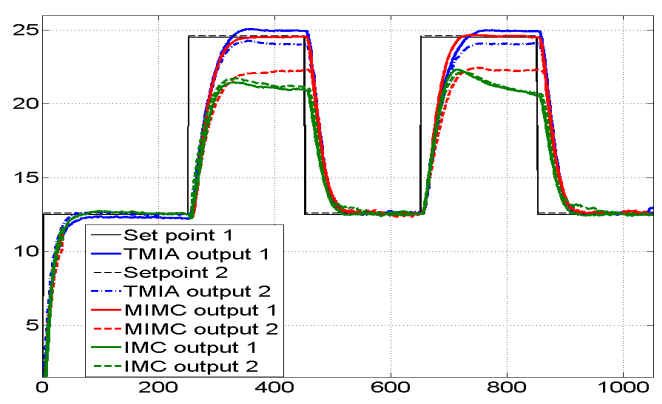

(a) Plant output Profile 1

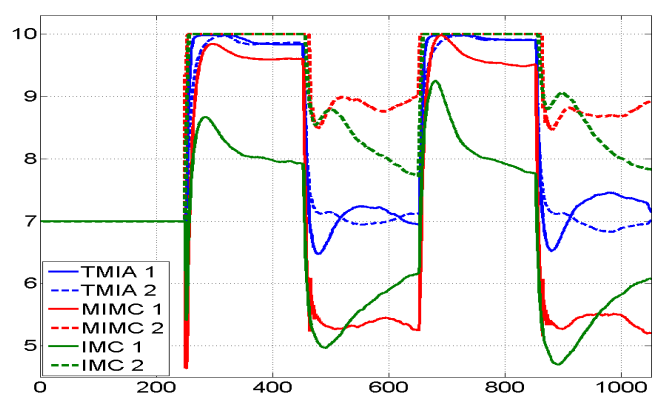

(b) Input voltages Profile 1

Fig. 5: Controller comparisons for Profile 1

2) Profile 2: In Figure 6a, both IMC and MIMC controllers are clearly unable to handle directionality problems in the Quadruple Tank system, their control inputs in Figure 6b remain saturated for a long time and steers the output of Tank 1 in the wrong direction. The TMIA controller handles the problem of directionality by recalculating optimal setpoints and control inputs which though much lower, steer the strongly coupled plant in the right direction. The inputs as seen in Figure $6 \mathrm{~b}$ remain unsaturated.

\section{B. PLC Implementation Results}

The TMIA program utilized only $19 \%(36.2 \mathrm{~KB})$ of the PLC working memory, $10 \%$ of its available RAM and $0.12 \%$ of retentive memory. $25 \%$ of this total utilized memory is contained in the two quadratic programs. Each interior point algorithm utilized 186 FLOPs including move operations. The highest number of iterations for the quadratic program is 37 as shown in Figure 7, the number of iterations tends to increase as the set-point requested increases and vice versa. The longest cycle time for the execution of the TMIA program obtained from the PLC online diagnostics was $30 \mathrm{~ms}$ which is far below the scan cycle monitoring rate of $1 \mathrm{~s}$. Thus the optimal solutions for the references and control inputs were always obtained during each scan cycle. These results indicate the the PLC can be used for the control of faster 


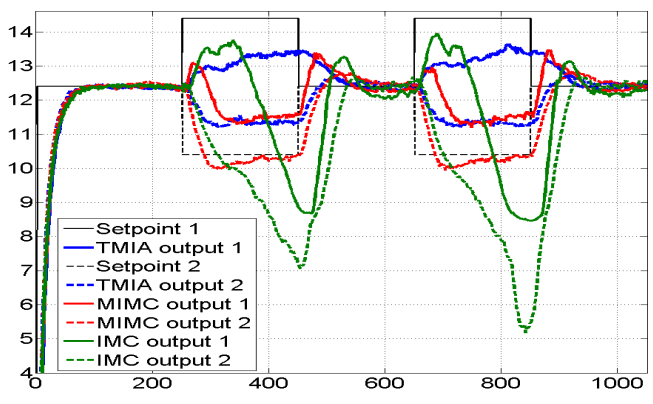

(a) Plant output Profile 2

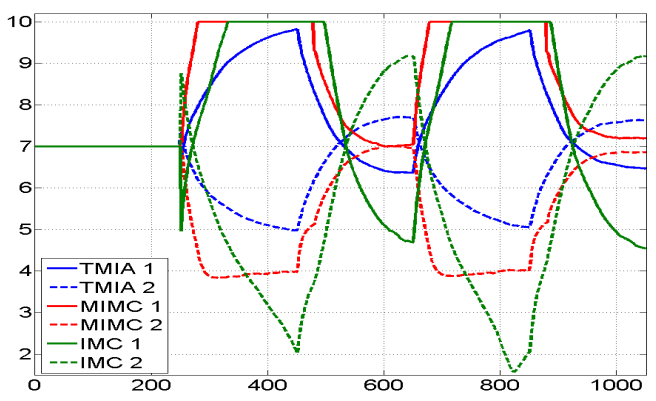

(b) Input voltages Profile 2

Fig. 6: Controller comparisons for Profile 2

and more complex processes. From the plots in Figure 7 it is seen that there is a direct relationship between the number of iterations and the execution time.

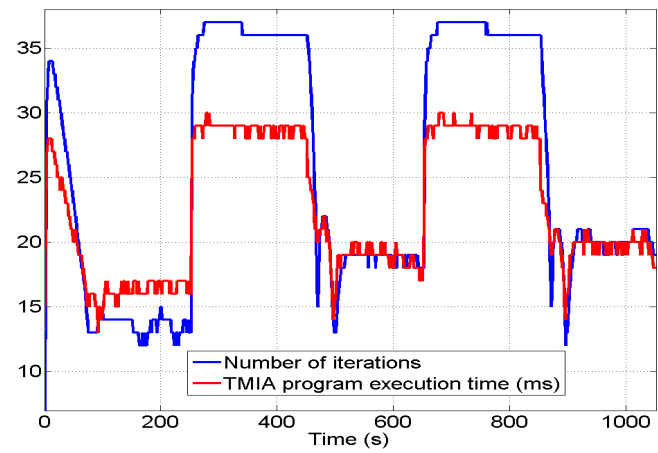

Fig. 7: Number of iterations (Interior point algorithm) and TMIA program execution time for profile 1.

\section{CONCLUSIONS}

The TMIA controller was successfully implemented on the Quadruple Tank system via a Siemens SIMATIC S7300 CPU 314C-2 PN/DP PLC and the Quanser QuaRC platform. The TMIA structure in comparison was found to outperform the classical IMC and Modified IMC controllers in terms of handling windup and directionality when extreme set-points are requested. From the memory utilization and execution time of the TMIA controller, a standard industrial
PLC has been shown to be a cost effective solution for the implementation of an advanced control technique without the need for an expensive and specialized system. This is beneficial in an industrial setting since PLCs provide the stability and reliability which most digital computers do not offer. The TMIA controller is shown to be a useful tool for the practical implementation of optimized multivariable control as it offers the advantages of single horizon computation requirements as compared to MPC.

\section{ACKNOWLEDGEMENTS}

Funding from Petroleum Technology Development Fund (PTDF) is greatly appreciated.

\section{REFERENCES}

[1] C. Edwards and I. Postlethwaite, "Anti-windup and bumpless-transfer schemes," Automatica, vol. 34, no. 2, pp. 199-210, 1998.

[2] A. Zheng, M. V. Kothare, and M. Morari, "Anti-windup design for internal model control," International Journal of Control, vol. 60, no. 5, pp. 1015-1024, 1994.

[3] P. Campo and M. Morari, "Robust control of processes subject to saturation nonlinearities," Computers and Chemical Engineering, vol. 14, no. 4, pp. 343-358, 1990.

[4] Y. Peng, D. Vrani, R. Hanus, and S. WELLER SR, "Anti-windup designs for multivariable controllers," Automatica, vol. 34, no. 12, pp. 1559-1565, 1998.

[5] M. Morari, Robust process control. Morari, 1989.

[6] V. Kapila and K. Grigoriadis, Actuator saturation control. CRC Press, 2002.

[7] M. Soroush and S. Valluri, "Optimal directionality compensation in processes with input saturation non-linearities," International Journal of Control, vol. 72, no. 17, pp. 1555-1564, 1999.

[8] T. A. Johansen and T. I. Fossen, "Control allocation: A survey," Automatica, vol. 49, no. 5, pp. 1087-1103, 2013.

[9] S. J. Qin and T. A. Badgwell, "A survey of industrial model predictive control technology," Control engineering practice, vol. 11, no. 7, pp. 733-764, 2003.

[10] Y. Wang and S. Boyd, "Fast model predictive control using online optimization," Control Systems Technology, IEEE Transactions on, vol. 18, no. 2, pp. 267-278, 2010.

[11] B. Huyck, H. J. Ferreau, M. Diehl, J. De Brabanter, J. F. Van Impe, B. De Moor, and F. Logist, "Towards online model predictive control on a programmable logic controller: practical considerations," Mathematical Problems in Engineering, vol. 2012, 2012.

[12] B. Huyck, J. De Brabanter, B. De Moor, J. Van Impe, and F. Logist, "Model predictive control of a pilot-scale destination column using a programmable automation controller,' in European Control Conference, 2013, Conference Proceedings, pp. 1053-1058.

[13] G. Valencia-Palomo and J. Rossiter, "Programmable logic controller implementation of an auto-tuned predictive control based on minimal plant information," ISA transactions, vol. 50, no. 1, pp. 92-100, 2011.

[14] N. Instruments, "A comparison of pacs to plcs," http://www.ni.com/ white-paper/2960/en/, accessed March 1, 2014.

[15] A. A. Adegbege and W. P. Heath, "Internal model control design for input constrained multivariable processes," AIChE Journal, vol. 57, no. 12, pp. 3459-3472, 2011.

[16] K. H. Johansson, "The quadruple-tank process: A multivariable laboratory process with an adjustable zero," Control Systems Technology, IEEE Transactions on, vol. 8, no. 3, pp. 456-465, 2000.

[17] C. E. Garcia and M. Morari, "Internal model control. 2. design procedure for multivariable systems," Industrial and Engineering Chemistry Process Design and Development, vol. 24, no. 2, pp. 472-484, 1985.

[18] Quanser, "Coupled water tanks," no. 5. [Online]. Available: http://www.yarbis.yildiz.edu.tr/web/userCourseMaterials/yeren ccb0e28fbb55c905118e131dca10026c.pdf

[19] S. Galeani, S. Tarbouriech, M. Turner, and L. Zaccarian, "A tutorial on modern anti-windup design," European Journal of Control, vol. 15, no. 3, pp. 418-440, 2009

[20] J. Nocedal and S. Wright, "Numerical optimization, series in operations research and financial engineering," Springer, New York, 2006. 